\title{
Predictors associated with early and late restenosis of arteriovenous fistulas and grafts after percutaneous transluminal angiography
}

\author{
Quan Zheng ${ }^{1,2}$, Bingying Xie ${ }^{1,2}$, Xinfu Xie ${ }^{1,3}$, Wenhao Zhang ${ }^{1,2}$, Jihua Hou ${ }^{1,3}$, Zhonglin Feng ${ }^{1}$, Yiming Tao ${ }^{1}$, \\ Feng Yu ${ }^{1}$, Li Zhang ${ }^{1}$, Zhiming Ye ${ }^{1}$ \\ ${ }^{1}$ Department of Nephrology, Guangdong Provincial People's Hospital (Guangdong Academy of Medical Sciences), Guangzhou, China; ${ }^{2}$ Department \\ of Nephrology, Shantou University Medical College, Shantou, China; ${ }^{3}$ Department of Nephrology, Second School of Clinical Medicine, Southern \\ Medical University, Guangzhou, China. \\ Contributions: (I) Conception and design: Z Ye, Q Zheng; (II) Administrative support: Z Ye; (III) Provision of study materials or patients: Z Ye, \\ Z Feng, Y Tao, F Yu, Q Zheng; (IV) Collection and assembly of data: Q Zheng, B Xie, X Xie, W Zhang, J Hou; (V) Data analysis and interpretation: \\ Q Zheng, Z Ye, L Zhang; (VI) Manuscript writing: All authors; (VII) Final approval of manuscript: All authors. \\ Correspondence to: Zhiming Ye. Department of Nephrology, Guangdong Provincial People's Hospital (Guangdong Academy of Medical Sciences), \\ Guangzhou 510080, China. Email: yzmgz@139.com.
}

Background: The objective of this study was to assess postintervention patency and analyze the predictive factors associated with early and late restenosis after intervention in hemodialysis arteriovenous fistulas (AVF) and arteriovenous grafts (AVG).

Methods: This study retrospectively analyzed 284 hemodialysis patients who underwent percutaneous transluminal angioplasty (PTA) due to AVF and AVG stenosis. A total of 350 PTA procedures were performed. Clinical, anatomical, biochemical, and technical variables were analyzed. Using univariate and multivariate analyses, we assessed the postintervention patency of PTA by follow-up, and identified the predictive factors taking into account competing risks.

Results: Postintervention patency rates at 3, 6, 12, and 24 months were $86.5 \%, 66.4 \%, 42.6 \%$, and 29.8\%, respectively, with a median patency duration of $11 \pm 0.71$ months. Kaplan-Meier analysis showed that the patency rate of the AVF group ( $\mathrm{n}=271)$ was dramatically higher than the AVG group $(\mathrm{n}=79)$ at 3, 6, and 12 months after PTA, respectively (88.9\% vs. $78.5 \%$, 69.0\% vs. $57.4 \%, 48.8 \%$ vs. $20.0 \%, \mathrm{P}<0.01$ ). Cox survival analysis revealed that the factors associated with postintervention patency of AVF included age of fistulas, serum albumin (ALB) levels, location of stenoses, lesion length longer than $2 \mathrm{~cm}$, multiple stenoses, and maximal pressure of dilatation lower than $16 \mathrm{~atm}$. In addition, factors related to postintervention patency of AVG included the presence of diabetes and hypertension, and serum ALB.

Conclusions: This study demonstrated that the risk factors associated with postintervention patency of AVF included age of fistulas, lower levels of serum ALB, location of stenoses, lesion length longer than $2 \mathrm{~cm}$, multiple stenoses, and maximal pressure of dilatation lower than $16 \mathrm{~atm}$. In addition, risk factors related to postintervention patency of AVG included the presence of diabetes and lower levels of serum ALB, while the presence of hypertension was found to be a protective factor for reducing patency loss of AVG. Among all these factors, serum ALB and multiple stenoses tended to predict early restenosis, while pressure of dilatation tended to predict late restenosis.

Keywords: Hemodialysis; percutaneous transluminal angioplasty; vascular access; stenosis

Submitted Oct 31, 2020. Accepted for publication Jan 06, 2021.

doi: $10.21037 / \mathrm{atm}-20-7690$

View this article at: http://dx.doi.org/10.21037/atm-20-7690 


\section{Introduction}

Vascular access complications are the leading cause of hospitalization in hemodialysis (HD) patients, accounting for $15-25 \%(1,2)$. Early detection and treatment of vascular access dysfunction is related to enhanced survival and quality of life for HD patients. Arteriovenous fistulas (AVF) are regarded as the first choice for long-term vascular access due to their superior longevity and lower complication rates compared with arteriovenous grafts (AVG) and central venous catheters (3). Nonetheless, vascular stenosis is the most common cause of AVF and AVG dysfunction and failure, often leading to thrombosis and occlusion (4).

In recent years, percutaneous transluminal angioplasty (PTA) has been recommended as first-line therapy for vascular access stenosis and occlusion because it is safe, effective, and minimally invasive compared to surgical revision (3). PTA can restore the flow of occluded vascular vessels, with a success rate of $80-100 \%$ (5). And it is an established treatment for stenosis in both AVFs and AVGs, which can significantly extend the patency duration of fistulas $(6,7)$. However, as a result of recurrent stenosis, repeated interventions have often been required, with a reported average of 3.1-3.5 times before access is ultimately abandoned $(5,6)$. The durability of PTA is limited, with a 12-month postintervention patency rate ranging between 26$64 \%(8,9)$. Variables that can affect patency after PTA have been studied in several previous reports, including clinical, anatomical, technical, and biochemical factors, however, conclusions were inconsistent or even contradictory $(6,10-12)$. Furthermore, there have been few reports on the factors influencing restenosis of AVF and AVG respectively. Despite these previous studies, the predictive factors associated with either early restenosis or late restenosis remain unknown. The ability to predict outcomes of PTA will allow closer monitoring of patients, and establishment of proper therapeutic standards for PTA and surgery. The aims of this study were therefore to evaluate patency after PTA with 3 -year follow-up, and analyze the various predictive factors associated with early and late restenosis after intervention in HD AVF and AVG. We present the following article in accordance with the STROBE reporting checklist (available at http://dx.doi.org/10.21037/atm-20-7690).

\section{Methods}

\section{Patient population}

The records of $284 \mathrm{HD}$ patients (143 men, $59 \pm 14$ years) who had undergone a successful PTA due to vascular access stenosis in Guangdong Provincial People's Hospital between August 2016 and September 2019 were retrospectively reviewed. In total, 350 interventions were performed on the patients, $26 \%$ of whom required multiple subsequent PTAs (twice on 43 patients, 3 times on 27 patients, and 4 times on 1 patient). Furthermore, 271 interventions were performed for AVF stenosis (70.6\%), and 79 interventions were performed for AVG stenosis (29.4\%). This study was approved by Guangdong Provincial People's Hospital Research Ethics Committee (No. GDREC 2020170H). All patients signed a consent form for treatment, and our institutional review board approved the study protocols. The study conformed to the provisions of the Declaration of Helsinki (as revised in 2013).

The patients received regular blood tests as part of routine care after admission to hospital. PTA was indicated for patients with a $\geq 50 \%$ stenosis or a short segment thrombosis and one or more of the following clinical abnormalities: (I) blood flow rate decreased to $<200 \mathrm{~mL} / \mathrm{min}$ by sonographic dilution (Mindray M9.0, Mindray Medical Corp, Shenzhen, China), (II) venous pressure increased to $>150 \mathrm{mmHg}$ during dialysis, (III) unexplained reduction of dialysis efficiency, (IV) an abnormal physical condition (decreased thrill, limb swelling, difficult cannulation, and prolonged bleeding from puncture sites). Patients with serious heart failure, cerebrovascular disease, or cancer were excluded from our study.

\section{Endovascular procedures}

Diagnostic fistulography or B-ultrasound of the AVF was performed after puncture of the outflow vein. When a stenotic or occlusive lesion was detected, a balloon catheter was then advanced to the lesion, and the balloon was inflated until technical success. Balloon diameters of 3-7 mm were chosen by visual estimation of the diameter of the adjacent non-stenotic vessel, and the balloon diameter was oversized by $1 \mathrm{~mm}$ in comparison. The balloons used in this study were high pressure balloons with a working pressure of 8-10 atm and a blasting pressure of 24-30 atm. Inflation was maintained at a pressure of 8-24 atm for $30 \mathrm{~s}$ each time until full expansion of the balloon was achieved based on operator experience. Heparin was used with doses ranging 2,000-12,000 IU during the procedure, and all patients were monitored with pulse oximetry, electrocardiography, and blood pressure determination. Technical success was defined as a residual stenosis $\leq 30 \%$ after the procedure. 
Clinical success was defined as the ability to provide adequate blood flow for dialysis. Only successful PTAs were included for analysis in this study.

\section{Definitions and study endpoints}

Variables were collected after the interventions, including clinical, biochemical, anatomical, and technical data. The clinical variables included age ( $<65, \geq 65$ years), primary disease (presence of diabetes or hypertension), durations of dialysis, age of fistulas, and use of antiplatelet and anticoagulant agents. The biochemical variables which were assayed routinely before the intervention included hemoglobin $(\mathrm{Hb})$, albumin (ALB), serum calcium, serum phosphate, calcium-phosphate product, parathyroid hormone (PTH), serum cholesterol (CHOL), low density lipoprotein (LDL), D-dimer, homocysteine (HCY), and C-reactive protein (CRP). Anatomical variables consisted of type of access (AVF or AVG), length of stenosis ( $\leq 2 \mathrm{~cm}$, $>2 \mathrm{~cm})$, presence of occlusion, degree of stenosis $(\leq 80 \%$, $>80 \%$ ), number of stenoses (single or multiple), and location of stenosis. Technical variables included the operation method [guided by B-ultrasound or digital angiography (DSA)], time of PTA, balloon diameter ( $\leq 6 \mathrm{~mm},>6 \mathrm{~mm}$ ), times of dilatations $(\leq 3,>3)$, maximal pressure of dilatations $(<16 \mathrm{~atm}, \geq 16 \mathrm{~atm})$, and residual stenosis after intervention $(<15 \%, \geq 15 \%)$.

In the case of multiple stenoses, cumulative lesion length was recorded. The degree of stenosis before PTA was expressed as a percentage and was defined as the narrowest diameter compared with the adjacent non-stenotic vessel immediately upstream of the target lesion. The location of the AVF stenosis was designated as: (I) anastomotic and peri-anastomotic (the first $2 \mathrm{~cm}$ of the vein distally to the anastomosis), (II) venous outflow stenosis (the location for puncture in the forearm and elbow), (III) upper arm and central vein stenosis, (IV) arterial (13). Central vein stenoses were defined as those located in the subclavian or brachiocephalic vein or superior vena cava. For multiple lesions, we only recorded the location of lesions with a greater degree of stenosis. Primary patency after PTA was defined as the time from the initial PTA until the second PTA, vascular access failure, or study end, whichever occurred first. Secondary patency after PTA was defined as the time from the initial PTA until the abandonment of the vascular access or study end, regardless of the number of subsequent PTAs (13). Early restenosis was defined as the recurrence of stenosis within 3 months after PTA, and late restenosis was defined as the recurrence of stenosis beyond 6 months in this study.

Follow-up included clinical examinations, measurement of vascular access venous pressure during dialysis performed every month after PTA, and measurement of the vascular access flow with ultrasonography performed every 3 months or if there was any clinical evidence of significant restenosis. Follow-up of the patient cohort after the first intervention ranged from 6 to 36 months. Patients who died, underwent transplantation, or were lost to follow-up were also considered endpoints of the study.

\section{Statistical analysis}

Continuous variables were expressed as the mean \pm standard deviation, whereas categorical variables were expressed as frequencies or percentages. The underlying distributions of continuous variables were assessed by the Shapiro-Wilk test for normality. Comparisons were made by a student's t-test for normally distributed continuous variables, and the Wilcoxon rank sum test was used for skewed continuous variables. Categorical variables were compared using a chisquared test or Fisher's exact test. Patency curves were calculated using the Kaplan-Meier method and compared by the log-rank test. Multivariate analysis by the Cox proportional hazards model, using a backwards approach, was performed to highlight the independent association of studied variables with the patency of PTA. Candidate risk factors with $\mathrm{P}<0.2$ in the univariate analysis were included in the multivariate model (14). All reported $\mathrm{P}$ values were 2 -tailed, and $\mathrm{P}<0.05$ was considered statistically significant. Statistical analysis was conducted using SPSS for Windows (Version 24.0; IBM Corp, Armonk, NY.).

\section{Results}

\section{Population characteristics}

A total of $284 \mathrm{HD}$ patients were enrolled in this study. There was a preponderance of men $(n=152,53.8 \%)$. The mean age of the cohort was $59 \pm 14$ years (range, 21-91 years), and the mean vascular access age was $30 \pm 35$ months. The primary diseases of the patients included chronic glomerulonephritis $(\mathrm{n}=133,46.8 \%)$, diabetes mellitus ( $\mathrm{n}=88,30.9 \%)$, hypertensive kidney disease $(n=18,6.4 \%)$, polycystic kidney disease $(n=13,4.6 \%)$, and others $(n=32,11.3 \%)$. There were 350 endovascular interventions performed in the patients, with 271 PTAs 

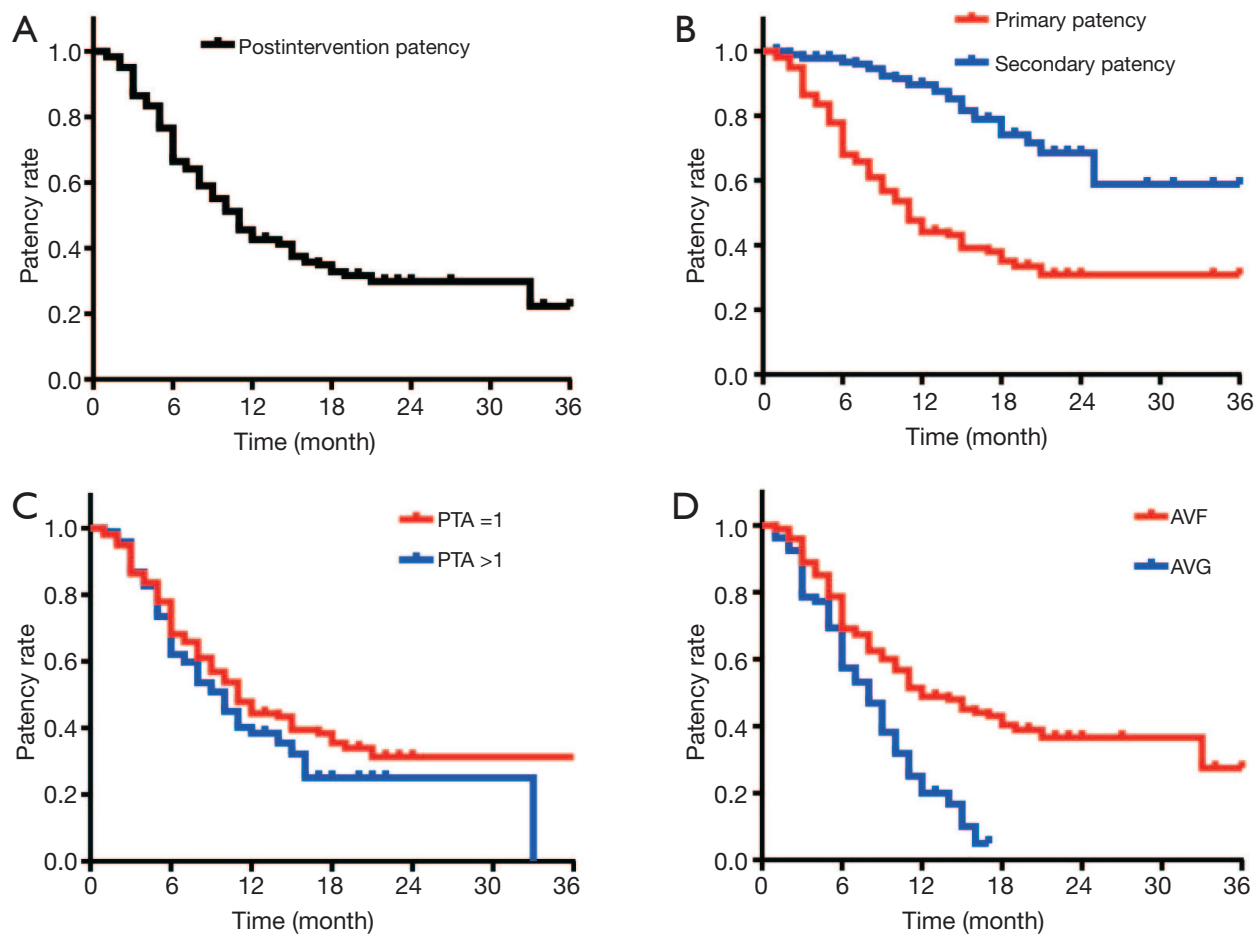

Figure 1 Kaplan-Meier estimates of postintervention patency after successful percutaneous transluminal angioplasty (PTA). (A) Patency of all interventions; (B) primary patency and secondary patency; $(\mathrm{C})$ the influence of PTA frequency on postintervention patency $(\mathrm{P}=0.016)$; $(\mathrm{D})$ postintervention patency of the arteriovenous fistula (AVF) and arteriovenous graft (AVG) groups $(\mathrm{P}<0.01)$.

performed for AVF stenosis (787.4\%) and 79 PTAs performed for AVG stenosis (22.6\%). A total of $25 \%$ of patients underwent multiple interventions ( 2 in 43 patients, 3 in 27 patients, and 4 in 1 patient). The mean length of stenoses was $3.79 \mathrm{~cm}$ (range, $0.2-13 \mathrm{~cm}$ ), and the mean degree was $90 \%$ (range, 50-100\%). The mean balloon diameter was $5.9 \pm 1.41 \mathrm{~mm}$ (range, $3-14 \mathrm{~mm}$ ), and the mean pressure and times of dilatations was $16.01 \pm 3.66 \mathrm{~atm}$ (range, 8-24 atm) and 4.1 \pm 1.92 times (range, 3-12 times), respectively.

\section{Postintervention patency}

The postintervention cumulative patency curve in the 350 PTAs are demonstrated in Figure 1, as determined by Kaplan-Meier analysis. The patency rate of all interventions was $86.5 \%$ at 3 months, $66.4 \%$ at 6 months, $42.6 \%$ at 12 months, and $29.8 \%$ at 24 months, and the median patency duration was $11 \pm 0.71$ months (Figure $1 A$ ). The primary patency rates at $3,6,12$, and 24 months were $86.4 \%, 68.0 \%, 44.0 \%$, and $30.8 \%$, respectively, and the secondary patency rates were $97.7 \%, 96.5 \%, 89.6 \%$, and
$68.6 \%$, respectively (Figure 1B). There were no significant differences between the primary patency rate and postreintervention patency rate, which was marginally lower than the former $(86.4 \%$ vs. $86.7 \%$ at 3 months, $68.0 \%$ vs. $62.1 \%$ at 6 months, $44.0 \%$ vs. $38.4 \%$ at 12 months, and $30.8 \%$ vs. $25.1 \%$ at 24 months, $\mathrm{P}=0.16$; Figure $1 C$ ). Kaplan-Meier analysis revealed that the patency rates were dramatically higher in the AVF group than the AVG group $(88.9 \%$ vs. $78.5 \%$ at 3 months, $69.0 \%$ vs. $57.4 \%$ at 6 months, $48.8 \%$ vs. $20.0 \%$ at 12 months, $\mathrm{P}<0.01$; Figure 1D). The median patency duration of AVF was $12 \pm 1.63$ months, while the median patency duration of AVG was $8 \pm 0.92$ months.

\section{Univariate analysis}

The continuous variables and categorical variables associated with patency at 3 and 12 months were compared using a student's t-test (Table 1) and chi-squared test (Table 2), respectively. The 3 -month stenosis group in Table 1 consisted of patients who had an early restenosis within 3 months after PTA. The 12-month stenosis group consisted of patients who 
Table 1 Continuous variables associated with patency at 3 and 12 months compared by student's t-test

\begin{tabular}{|c|c|c|c|c|c|c|}
\hline Variables & \multicolumn{3}{|c|}{3 months } & \multicolumn{3}{|c|}{12 months } \\
\hline Age (years) & $59.03(14.04)$ & $59.48(14.03)$ & 0.84 & $55.91(14.81)$ & $61.59(12.41)$ & 0.01 \\
\hline Time on HD (years) & $3.51(3.28)$ & $3.51(3.54)$ & 0.99 & $3.57(3.64)$ & $2.89(2.51)$ & 0.21 \\
\hline Age of VA (months) & 30.05 (35.44) & 29.51 (34.61) & 0.92 & 34.84 (39.63) & $22.46(22.44)$ & 0.02 \\
\hline ALB (g/L) & $38.73(4.46)$ & $37.04(4.22)$ & 0.02 & $39.31(4.76)$ & $38.15(4.29)$ & 0.14 \\
\hline $\mathrm{Ca}(\mathrm{g} / \mathrm{dL})$ & $9.76(1.29)$ & $9.82(1.32)$ & 0.77 & $9.79(1.18)$ & $9.76(1.31)$ & 0.91 \\
\hline $\mathrm{P}(\mathrm{mmol} / \mathrm{L})$ & $2.03(0.69)$ & $2.01(0.64)$ & 0.82 & $2.07(0.61)$ & $2.08(0.75)$ & 0.91 \\
\hline Ca-P product & $59.09(20.87)$ & $57.29(19.17)$ & 0.61 & $61.48(19.58)$ & $60.29(22.15)$ & 0.75 \\
\hline LDL (mmol/L) & $2.87(0.89)$ & $2.93(1.04)$ & 0.72 & $2.94(0.92)$ & $2.68(0.69)$ & 0.09 \\
\hline D-Dimer ( $\mu \mathrm{g} / \mathrm{L})$ & $1,440.52(3,067.23)$ & $2,458.21(3,845.91)$ & 0.11 & $1,425.89(3,903.51)$ & $1,107.5(3,127.22)$ & 0.61 \\
\hline CRP (mg/L) & 8.44 (11.99) & $10.29(13.24)$ & 0.51 & $8.38(13.11)$ & $8.57(8.75)$ & 0.94 \\
\hline $\mathrm{HCY}(\mu \mathrm{mol} / \mathrm{L})$ & $24.13(10.78)$ & 32.81 (18.89) & 0.09 & $23.77(11.02)$ & 24.49 (12.37) & 0.81 \\
\hline
\end{tabular}

Data are shown as mean (SD). *, the 3-month stenosis group consisted of patients who had an early restenosis in the 1-3 months after PTA. The 12-month stenosis group consisted of patients who had a late restenosis in the 6-12 months after PTA. HD, hemodialysis; VA, vascular access; HB, hemoglobin; ALB, albumin; Ca, serum calcium; P, serum phosphate; Ca-P product, serum calcium-phosphate product; PTH, parathyroid hormone; CHOL, serum cholesterol; LDL, low density lipoprotein; HCY, homocysteine; CRP, C-reaction protein.

had a late restenosis 6-12 months after PTA. In this way, we were able to identify which factors were associated with early or late restenosis after PTA.

The student's t-test revealed that serum ALB was significantly higher in the 3 -month patency group than the stenosis group $(\mathrm{P}=0.02)$. Factors that were statistically significant determinants for 12-month patency included age, age of vascular access (VA), and $\mathrm{Hb}$. The patients of the 12-month stenosis group were significantly older than the patency group $(\mathrm{P}=0.01)$. Age of $\mathrm{VA}(\mathrm{P}=0.02)$ and $\mathrm{Hb}$ $(\mathrm{P}=0.01)$ were also higher in the 12 -month patency group than the stenosis group (Table 1).

The chi-squared test also indicated that the 3 - and 12 -month patency rates were dramatically higher in the AVF group than the AVG group $(\mathrm{P}<0.05)$. There was a significant difference in 3-month patency between the groups in terms of locations of stenoses $(\mathrm{P}=0.01)$. The presence of hypertension $(\mathrm{P}=0.02)$ and multiple stenoses $(\mathrm{P}=0.05)$ resulted in lower 3-month patency. In addition, the 12-month patency was markedly lower in the dilatation $<16 \mathrm{~atm}$ group than the dilatation $\geq 16$ atm group $(\mathrm{P}=0.04$; Table 2$)$.

\section{Multivariate analysis}

Candidate risk factors with $\mathrm{P}<0.2$ in the univariate analysis were included in the multivariate analysis using Cox proportional hazards model with a backwards approach (14). Because of the significant differences between the AVF and AVF groups, they underwent multivariate analysis respectively, and the outcomes were quite different (Table 3). Factors associated with lower AVF postintervention patency consisted of older age of VA (HR $=1.037,95 \%$ CI: $1.007-$ $1.068, \mathrm{P}=0.016$ ), lower level of serum ALB ( $\mathrm{HR}=0.744$, 95\% CI: $0.607-0.912, \mathrm{P}=0.004)$, length of stenosis longer than $2 \mathrm{~cm}(\mathrm{HR}=7.309,95 \% \mathrm{CI}: 1.789-29.864, \mathrm{P}=0.007)$, and multiple stenoses ( $\mathrm{HR}=6.332,95 \% \mathrm{CI}: 1.056-37.863$, $\mathrm{P}=0.043)$. On the other hand, pressure of dilatations $\geq 16$ atm group (HR $=0.054,95 \%$ CI: $0.008-0.360, \mathrm{P}=0.023$ ) was significantly higher in AVF postintervention patency than pressure of dilations $<16$ atm group. It was revealed that the location of stenosis, especially the outflow vein, was also related to higher AVF patency than anastomotic and peri-anastomotic stenoses (HR $=0.021,95 \%$ CI: 0.003 - 
Table 2 Categorical variables associated with patency at 3 and 12 months compared by chi-squared test

\begin{tabular}{|c|c|c|c|c|}
\hline \multirow{2}{*}{ Variables [N.] } & \multicolumn{4}{|c|}{ Patency rate } \\
\hline & 3 months (\%) & $\mathrm{P}$ & 12 months $^{\mathrm{a}}(\%)$ & $P$ \\
\hline Diabetes & & 0.06 & & 0.33 \\
\hline Yes [129] & 89.1 & & 62.5 & \\
\hline No [221] & 82.2 & & 54.2 & \\
\hline Hypertension & & 0.02 & & 0.31 \\
\hline Yes [282] & 77.9 & & 50.0 & \\
\hline No [68] & 88.7 & & 61.5 & \\
\hline Antiplatelet or anticoa & agulant & 0.39 & & 0.59 \\
\hline Yes [125] & 87.9 & & 51.4 & \\
\hline No [101] & 86.3 & & 57.1 & \\
\hline VA types & & 0.02 & & $<0.01$ \\
\hline AVF [271] & 88.9 & & 66.4 & \\
\hline AVG [79] & 78.5 & & 29.6 & \\
\hline Location of stenosis $^{b}$ & & 0.01 & & 0.81 \\
\hline I [151] & 92.7 & & 62.5 & \\
\hline II [104] & 83.7 & & 67.3 & \\
\hline III [20] & $16 / 20^{\circ}$ & & $4 / 5^{c}$ & \\
\hline IV [8] & $3 / 8^{c}$ & & $1 / 4^{c}$ & \\
\hline Length of stenosis & & 0.38 & & 0.45 \\
\hline$\leq 2 \mathrm{~cm}[94]$ & 90.4 & & 62.0 & \\
\hline$>2$ cm [166] & 86.7 & & 68.5 & \\
\hline Number of stenosis & & $<0.05$ & & 0.23 \\
\hline Single [222] & 89.2 & & 63.1 & \\
\hline Multiple [126] & 81.7 & & 53.1 & \\
\hline Occlusion & & 0.27 & & 0.99 \\
\hline Yes [53] & 82.7 & & 65.1 & \\
\hline No [216] & 88.4 & & 65.0 & \\
\hline Degree of stenosis & & 0.06 & & 0.89 \\
\hline$\leq 80 \%$ [118] & 91.5 & & 65.6 & \\
\hline$>80 \%$ [149] & 83.9 & & 64.5 & \\
\hline Operation guided by & & 0.11 & & 0.91 \\
\hline B-ultrasound [31] & 77.4 & & 60 & \\
\hline DSA [314] & 87.6 & & 61.7 & \\
\hline PTA frequency & & 0.95 & & 0.67 \\
\hline 1 [252] & 86.5 & & 60.9 & \\
\hline$>1$ [98] & 86.7 & & 57.1 & \\
\hline
\end{tabular}

Table 2 (continued)
Table 2 (continued)

\begin{tabular}{|c|c|c|c|c|}
\hline \multirow{2}{*}{ Variables [N.] } & \multicolumn{4}{|c|}{ Patency rate } \\
\hline & 3 months (\%) & $P$ & 12 months $^{\mathrm{a}}(\%)$ & $\mathrm{P}$ \\
\hline Balloon diameter & & 0.38 & & 0.16 \\
\hline$>6 \mathrm{~mm}[274]$ & 83.1 & & 47.8 & \\
\hline$\leq 6 \mathrm{~mm}[66]$ & 87.2 & & 63.5 & \\
\hline Times of dilatations & & 0.06 & & 0.66 \\
\hline$\leq 3[141]$ & 90.8 & & 62.3 & \\
\hline$>3[85]$ & 82.4 & & 66.7 & \\
\hline $\begin{array}{l}\text { Pressure of } \\
\text { dilatations }\end{array}$ & & 0.32 & & 0.04 \\
\hline$<16$ atm [105] & 89.5 & & 54.0 & \\
\hline$\geq 16$ atm [128] & 85.2 & & 73.1 & \\
\hline Residual stenosis & & 0.06 & & 0.36 \\
\hline$<15 \%$ [156] & 85.3 & & 69.2 & \\
\hline$\geq 15 \%[35]$ & 97.1 & & 57.9 & \\
\hline
\end{tabular}

$\mathrm{N}$ represents the number of interventions. ${ }^{a}$, the 12-month patency rate was calculated after removing the patients who had a restenosis after 1-6 months, so that it was higher than the cumulative patency with the Kaplan-Meier analysis. ${ }^{b}$, the location of the AVF stenosis was designated as (I) anastomotic and peri-anastomotic (the first $2 \mathrm{~cm}$ of the vein distally to the anastomosis), (II) venous outflow stenosis (the location for puncture in the forearm and elbow), (III) upper arm and central vein stenosis, (IV) arterial. For multiple lesions, we only recorded the location of lesions with a greater degree of stenosis. ${ }^{c}$, the number of patients with upper arm, central vein, and arterial stenosis was small, so it is represented as a ratio of patency number to all numbers. VA, vascular access; DSA, digital angiography; PTA, percutaneous transluminal angioplasty.

0.137, $\mathrm{P}=0.001$ ). As for AVG postintervention patency, a statistically significant association with increased risk of patency failure was observed for the presence of diabetes (HR $=1.959,95 \%$ CI: $1.020-3.760, \mathrm{P}=0.043)$. Moreover, the presence of hypertension (HR $=0.388,95 \%$ CI: $0.189-0.796$, $\mathrm{P}=0.01)$ and a higher level of serum ALB (HR $=0.892,95 \%$ CI: $0.809-0.983, \mathrm{P}=0.021$ ) was protective against the loss of patency of AVG.

\section{Discussion}

Vascular stenosis is one of the most common complications of AVF and AVG, which usually results in thrombosis and VA failure. It is widely believed that intimal hyperplasia (IH) is the principal pathological cause of vascular stenosis 
Table 3 Multivariate analysis of factors associated with AVF and AVG patency

\begin{tabular}{|c|c|c|c|}
\hline Variables & $\mathrm{P}$ & $\mathrm{HR}$ & $95 \% \mathrm{Cl}$ \\
\hline \multicolumn{4}{|l|}{ AVF } \\
\hline Age & 0.133 & 0.947 & $0.881-1.017$ \\
\hline Age of VA & 0.016 & 1.037 & $1.007-1.068$ \\
\hline Diabetes & 0.946 & 0.947 & $0.198-4.536$ \\
\hline Hypertension & 0.503 & 2.782 & $0.139-55.731$ \\
\hline ALB & 0.004 & 0.744 & $0.607-0.912$ \\
\hline PTH & 0.395 & 1.001 & $0.999-1.002$ \\
\hline $\mathrm{CHOL}$ & 0.596 & 1.532 & $0.317-7.406$ \\
\hline LDL & 0.149 & 0.196 & $0.021-1.789$ \\
\hline Location of stenosis & 0.001 & 0.021 & $0.003-0.137$ \\
\hline Length of stenosis & 0.007 & 7.309 & $1.789-29.864$ \\
\hline Occlusion & 0.918 & 1.16 & $0.076-19.944$ \\
\hline Multiple stenosis & 0.043 & 6.332 & $1.056-37.863$ \\
\hline Pressure of dilatations & 0.023 & 0.054 & $0.008-0.360$ \\
\hline Times of dilatations & 0.322 & 2.316 & $0.440-12.202$ \\
\hline \multicolumn{4}{|l|}{ AVG } \\
\hline Diabetes & 0.043 & 1.959 & $1.020-3.760$ \\
\hline Hypertension & 0.01 & 0.388 & $0.189-0.796$ \\
\hline Antiplatelet & 0.801 & 1.095 & $0.541-2.216$ \\
\hline $\mathrm{HB}$ & 0.304 & 1.011 & $0.99-1.031$ \\
\hline ALB & 0.021 & 0.892 & $0.809-0.983$ \\
\hline Balloon diameter & 0.248 & 0.665 & $0.333-1.328$ \\
\hline
\end{tabular}

Data are expressed as hazard ratios with standard error of coefficient and $\mathrm{P}$ values. HR, hazard ratios; $\mathrm{Cl}$, confidence interval; AVF, arteriovenous fistula; AVG, arteriovenous graft; VA, vascular access; PTH, parathyroid hormone; CHOL, serum cholesterol; LDL, low density lipoprotein; HCY, homocysteine; $\mathrm{HB}$, hemoglobin; ALB, albumin.

in AVF and AVG $(2,15)$. A series of upstream factors were identified to promote the development of $\mathrm{IH}$, including abnormal flow conditions after anastomosis, repeated puncture injuries during dialysis, trauma caused by surgery or PTA, and biocompatibility of grafts (15). Brahmbhatt et al. concluded that common pathways for IH involved inflammation, oxidative stress, hemodynamics, and uremia (2). In addition, several clinical studies reported other stenotic patterns, including vascular calcifications, shrinking lumen, and venous valve-related stenosis $(7,16,17)$.
Although PTA has provided an excellent option for vascular stenosis, it can also act as a trigger for restenosis. Understanding the predictors of patency after PTA is helpful for comprehending the pathogenesis of vascular stenosis and finding novel treatments.

The medium patency duration of AVFs and AVGs after PTA in the present study was $11 \pm 0.71$ months, which is comparable to the duration of $8-13$ months reported in the literature (8). The postintervention primary and secondary patency rates at 12 months were $44.0 \%$ and $89.6 \%$, respectively, which is also consistent with the rates in previous studies $(8,12,13)$. Owing to economic and local medical conditions, some patients gave up the repairable AVF or AVG, and chose a new fistula or central venous catheter instead of a PTA according to our follow-up. Several studies have reported on the predictors of primary and secondary patency, but the results were inconsistent as there was no well-defined criteria for the abandonment of VA $(11,12)$. Compared to AVG, dramatically higher postintervention patency of AVF was found in this study, in agreement with previous studies $(18,19)$. There have been a number of studies that have analyzed the factors associated with postintervention patency of AVF and AVG together $(6,20)$, however, few studies have investigated them separately. Thus, we studied the predictors related to patency of AVF and AVG respectively.

The clinical factors that were found to be related to the postintervention patency of AVF and AVG in this study included age of VA, and the presence of diabetes and hypertension. Multivariate analysis showed that older age of VA was associated with lower patency of AVF after PTA. However, the univariate analysis showed that the age of VA was younger in the stenosis group than the patency group at 12 months after PTA. Several previous studies have indicated the potential associations between fistula age and postintervention patency $(8,11,21,22)$. Neuen et al. reported that the patency rate was lower for VA younger than 6 months compared with those older than 6 months (11), and Heye et al. showed that restenosis occurred earlier in AVF created less than 1 year compared with those at least 1 year old (8). Furthermore, Higashiura et al. found that the patency rate of non-maturing fistulas (younger than 3 months) was worse than mature fistulas (12). In Neuen et al.'s opinion, fistulas with early stenosis were likely to be intrinsically faulty and had a tendency to form neointimal hyperplasia (23). The mean age of VA in this study was $30 \pm 35$ months, which was much older than the mean age of VA in previous studies $(8,11,21,22)$. The different results 
in our study raises the possibility that the relationship between age of VA and patency of AVF is U-shaped rather than linear. The effect of age of VA should therefore be considered exploratory, and requires further investigation with larger cohorts in randomized studies.

The influence of diabetes and hypertension on postintervention patency is controversial. Most studies have suggested that diabetes and hypertension are not associated with patency after PTA $(6,20,24)$, while some studies found that diabetes is an important risk factor for fistula restenosis $(13,25)$. Our result showed that diabetes was associated with lower patency of AVG, but had no relation to patency of AVF. Wu et al. found that high glucose itself could directly impair endothelial progenitor cells, thereby affecting vascular repair and promoting the formation of neointima. They concluded that metabolic alteration caused by diabetes may lead to a prothrombotic environment, endothelial dysfunction, dysregulation of growth factors, and extracellular matrix deposition, all of which may predispose patients to restenosis (26). The present study also found that hypertension was protective against the restenosis of AVG, especially early restenosis. Liu et al. reported that HD patients with hypertension had a better patency rate of AVF than those without hypertension (27), which was consistent with our study. This mechanism may be due to the relationship between higher blood pressure and higher blood flow, which is an identified positive predictor of fistula stenosis $(22,24)$. However, patients with hypertension still need treatment because of the damage to target organs. The target value of blood pressure needs to be determined in order to balance the relationship between blood pressure and blood flow. Another potential reason behind hypertension patients having lower postintervention patency may be due to the use of calcium channel blockers (CCBs), angiotensin-converting enzyme inhibitors (ACEIs), or angiotensin receptor blockers (ARBs). Most patients in our cohort were using or had used CCBs, ACEIs, or ARBs. A small study showed that the use of CCBs could significantly improve postintervention primary patency (28). Furthermore, previous studies have reported the association between ACEIs or ARBs with greater patency of AVF (29). Further prospective trials with larger cohorts are required to validate these findings.

In regards to biochemical factors, a lower level of serum ALB was identified to predict the early restenosis of AVF and AVG. Liu et al. reported that lower serum ALB was associated with a significantly poorer patency rate of AVF (27), however, a number of other studies documented that serum ALB had no connection with patency of AVF and AVG $(11,26,30)$. Serum ALB is accepted as an indicator of nutrition and inflammation. Liu et al. believed that the impact of lower serum ALB on restenosis was due to the effect of inflammation on the patency rate of AVF (27).

In terms of anatomical factors, we found that stenosis length, multiple stenoses, and location of stenoses were related to the postintervention patency of AVF rather than AVG. Multivariate analysis revealed the stenosis length longer than $2 \mathrm{~cm}$ predicted poorer patency of AVF after PTA, which is consistent with the most recent literature $(13,24,25,31)$. A study by Higashiura et al. suggested that longer lesion length was an important predictor of postintervention patency of both late and early dysfunctional AVF (12). Neuen et al.'s study indicated that the influence of stenosis length on postintervention patency was related to the location of the stenosis. They reported that lesion length was more important in inflow arteries and outflow veins (including the anastomotic site), but less influential in central vein stenosis (23). The presence of multiple stenoses compared with a single stenosis increased the risk of early restenosis of AVF in our study, which is concordant with a retrospective study by Neuen et al. (11). This association was intuitive, but other studies have documented that multiple stenoses have no association with restenosis of AVF and AVG $(13,24,25,32)$. The stenosis locations of the central vein $(n=20,7.1 \%)$ and arterial stenoses $(\mathrm{n}=8,2.8 \%)$ in our cohort accounted for a small proportion, while most of the stenoses were juxta-anastomotic ( $\mathrm{n}=151,53.4 \%)$ or outflow vein stenoses ( $\mathrm{n}=104,36.7 \%)$. According to our results, PTA was less effective in the treatment of juxta-anastomotic stenosis than outflow vein stenosis of AVF. While several studies have examined the effect of lesion location on postintervention patency, the results have not been consistent $(13,23,32)$. A number of studies indicated that anastomotic stenosis was a risk factor related to restenosis of AVF (12), while Romann et al. reported that outflow vein stenosis was associated with requiring a second PTA (13). Zhu et al.'s study suggested that inflow artery stenosis $(\mathrm{HR}=3.83$, 95\% CI: 1.46-10.09) and anastomosis (HR $=1.9,95 \% \mathrm{CI}$ : 1.09-3.32) were significant predictors of reduced patency of AVF (32). Classification comparison of lesion location was different, and stenosis location was defined variably among studies, especially in the case of multiple stenoses. These results therefore require confirmation with larger cohorts including lesions in various stenosis locations.

In terms of technical factors, only the pressure of 
dilatations was identified to be associated with late restenosis of AVF. The balloons used in this study were high pressure balloons with a working pressure of 8-10 atm and a blasting pressure of 24-30 atm. The mean maximal pressure of dilatations was $16.01 \pm 3.66 \mathrm{~atm}$ (range, $8-20 \mathrm{~atm}$ ), and the results showed that pressure of dilatation lower than $16 \mathrm{~atm}$ was associated with late restenosis of AVF in the present study. However, the results of Miyamoto et al.'s study indicated that the pressure of dilatation with a mean of $15 \pm 5.8 \mathrm{~atm}$ was unrelated to the postintervention patency of AVF (24). Zhu et al. reported that there was no significant difference in long-term patency between high pressure balloons and plain balloons (32). Similarly, Wakamoto et al.'s study also documented that the 3- and 12-month patency showed no obvious differences between the low pressure $(8 \mathrm{~atm})$ group and the high pressure (30 atm) group (33). In our opinion, a certain increased pressure of dilatation may reduce the loss of patency by achieving residual stenosis much less than $30 \%$, which is associated with postintervention patency according to previous studies $(25,32)$. The potential association between several technical factors and postintervention patency such as balloon diameter, pressure and times of dilatation, and duration of inflation remains largely undetermined. Elramah et al.'s study indicated that there was a clear advantage for shorter inflation duration of $30 \mathrm{~s}$ in 3 -month patency, compared with an inflation duration of 1 minute (9). Moreover, Zhu et al. suggested that dilatation more than 2 times was a significant predictor of restenosis of AVF after PTA (32). The effects of the abovementioned technical factors could explain the differences between our results and those of other studies.

The relationship between postintervention patency and the use of antiplatelet and anticoagulant agents has also been studied $(11,20,24)$, however, no significant relationship was observed in this study. Lin et al. (34) reported that the use of aspirin $(\mathrm{P}<0.0001)$, clopidogrel $(\mathrm{P}=0.03)$, and warfarin $(\mathrm{P}=0.0002)$ could dramatically decrease the primary patency of AVF. Furthermore, Kim et al.'s study found that the use of antiplatelet agents before, during, and after PTA was associated with reduced secondary patency (20). However, most of the previous studies have shown that the use of antiplatelet and anticoagulant medications were not necessary to improve the proportion of AVF $(11,24)$. In this study, the decision to use antiplatelet and anticoagulant agents for some patients was based on their complications, such as coronary heart disease, atrial fibrillation, and cerebral infarction, regardless of PTA. This may have resulted in selection bias, and future randomized controlled trials are required to explore the necessity of antiplatelet and anticoagulant agents after PTA.

This study was a retrospective analysis and a single center study, with inherent drawbacks of such approaches. However, this center is a tertiary referral hospital and serves a large provincial population from both local and outside regions. We could not exclude the possibility that unmeasured confounding factors which have been speculated to be associated with postintervention patency might have influenced our results, such as the type of AVF, blood flow after PTA, and the use of CCBs, ACEIs, or ARBs. As with any observational study, we were unable to conclude causal relationships, but through comprehensive data collection and robust statistical methodology, we were able to find associations between various predictive factors and early or late restenosis of AVF and AVG.

In conclusion, our results demonstrated that the risk factors associated with postintervention patency of AVF included age of fistulas, lower levels of serum ALB, location of stenoses, lesion length longer than $2 \mathrm{~cm}$, multiple stenoses, and maximal pressure of dilatation lower than 16 atm. In addition, risk factors related to postintervention patency of AVG included the presence of diabetes and lower levels of serum ALB, while the presence of hypertension was found to be a protective factor for reducing patency loss of AVG. Among all these factors, serum ALB and multiple stenoses tended to predict early restenosis, while pressure of dilatation tended to predict late restenosis. Further studies are required to investigate the effects of fistula age and stenosis location on postintervention patency.

\section{Acknowledgments}

Funding: This study was supported by the Natural Science Foundation of Guangdong Province (Grants 2018A0303130336; 2020A1515010137) and Scientific Research Project of Guangdong Provincial People's HospitalSummit Plan (Grants KJ012019436; DFJH201911).

\section{Footnote}

Reporting Checklist: The authors have completed the STROBE reporting checklist. Available at http://dx.doi. org/10.21037/atm-20-7690

Data Sharing Statement: Available at http://dx.doi. org/10.21037/atm-20-7690 
Conflicts of Interest: All authors have completed the ICMJE uniform disclosure form (available at http://dx.doi. org/10.21037/atm-20-7690). The authors have no conflicts of interest to declare.

Etbical Statement: The authors are accountable for all aspects of the work in ensuring that questions related to the accuracy or integrity of any part of the work are appropriately investigated and resolved. This study was approved by Guangdong Provincial People's Hospital Research Ethics Committee (No. GDREC 2020170H). All patients signed a consent form for treatment, and our institutional review board approved the study protocols. The study conformed to the provisions of the Declaration of Helsinki (as revised in 2013)

Open Access Statement: This is an Open Access article distributed in accordance with the Creative Commons Attribution-NonCommercial-NoDerivs 4.0 International License (CC BY-NC-ND 4.0), which permits the noncommercial replication and distribution of the article with the strict proviso that no changes or edits are made and the original work is properly cited (including links to both the formal publication through the relevant DOI and the license). See: https://creativecommons.org/licenses/by-nc-nd/4.0/.

\section{References}

1. Collins AJ, Foley RN, Chavers B, et al. 'United States Renal Data System 2011 Annual Data Report: Atlas of chronic kidney disease \& end-stage renal disease in the United States. Am J Kidney Dis 2012;59:A7, e1-420.

2. Brahmbhatt A, Remuzzi A, Franzoni M, et al. The molecular mechanisms of hemodialysis vascular access failure. Kidney Int 2016;89:303-16.

3. National Kidney Foundation. KDOQI Clinical Practice Guideline for Hemodialysis Adequacy: 2015 update. Am J Kidney Dis 2015;66:884-930.

4. Riella MC, Roy-Chaudhury P. Vascular access in haemodialysis: strengthening the Achilles' heel. Nat Rev Nephrol 2013;9:348-57.

5. Balamuthusamy S, Reddi AL, Madhrira MH, et al. Clinical predictors of recurrent stenosis and need for re-intervention in the cephalic arch in patients with brachiocephalic AV fistulas. J Vasc Access 2017;18:319-24.

6. Malka KT, Flahive J, Csizinscky A, et al. Results of repeated percutaneous interventions on failing arteriovenous fistulas and grafts and factors affecting outcomes. J Vasc Surg 2016;63:772-7.

7. Suemitsu K, Shiraki T, Iida O, et al. Impact of Lesion Morphology on Durability After Angioplasty of Failed Arteriovenous Fistulas in Hemodialysis Patients. J Endovasc Ther 2018;25:649-54.

8. Heye S, Maleux G, Vaninbroukx J, et al. Factors influencing technical success and outcome of percutaneous balloon angioplasty in de novo native hemodialysis arteriovenous fistulas. Eur J Radiol 2012;81:2298-303.

9. Elramah M, Boujelbane L, Yevzlin AS, et al. Dialysis access venous stenosis: treatment with balloon angioplasty 30-second vs. 1-minute inflation times. Hemodial Int 2015;19:108-14.

10. Mortamais J, Papillard M, Girouin N, et al. Endovascular treatment of juxta-anastomotic venous stenoses of forearm radiocephalic fistulas: long-term results and prognostic factors. J Vasc Interv Radiol 2013;24:558-64; quiz 565.

11. Neuen BL, Gunnarsson R, Baer RA, et al. Factors associated with patency following angioplasty of hemodialysis fistulae. J Vasc Interv Radiol 2014;25:1419-26.

12. Higashiura W, Takara H, Kitamura R, et al. Factors Associated with Secondary Functional Patency After Percutaneous Transluminal Angioplasty of the Early Failing or Immature Hemodialysis Arteriovenous Fistula. Cardiovasc Intervent Radiol 2019;42:34-40.

13. Romann A, Beaulieu MC, Rhéaume P, et al. Risk factors associated with arteriovenous fistula failure after first radiologic intervention. J Vasc Access 2016;17:167-74.

14. Rajan DK, Bunston S, Misra S, et al. Dysfunctional autogenous hemodialysis fistulas: outcomes after angioplasty--are there clinical predictors of patency? Radiology 2004;232:508-15.

15. Duque JC, Tabbara M, Martinez L, et al. Dialysis Arteriovenous Fistula Failure and Angioplasty: Intimal Hyperplasia and Other Causes of Access Failure. Am J Kidney Dis 2017;69:147-51.

16. Jankovic A, Damjanovic T, Djuric Z, et al. Impact of vascular calcifications on arteriovenous fistula survival in hemodialysis patients: a five-year follow-up. Nephron 2015;129:247-52.

17. Jankovic A, Damjanovic T, Djuric Z, et al. Calcification in arteriovenous fistula blood vessels may predict arteriovenous fistula failure: a 5-year follow-up study. Int Urol Nephrol 2017;49:881-7.

18. Ravani P, Palmer SC, Oliver MJ, et al. Associations between hemodialysis access type and clinical outcomes: a systematic review. J Am Soc Nephrol 2013;24:465-73.

19. Yan Y, Clark TW, Mondschein JI, et al. Outcomes of 
percutaneous interventions in transposed hemodialysis fistulas compared with nontransposed fistulas and grafts. J Vasc Interv Radiol 2013;24:1765-72; quiz 1773.

20. Kim SM, Ko HK, Noh M, et al. Factors Affecting Patency following Successful Percutaneous Intervention for Dysfunctional Hemodialysis Vascular Access. Ann Vasc Surg 2018;47:54-61.

21. Maeda K, Furukawa A, Yamasaki M, et al. Percutaneous transluminal angioplasty for Brescia-Cimino hemodialysis fistula dysfunction: technical success rate, patency rate and factors that influence the results. Eur J Radiol 2005;54:426-30.

22. Heerwagen ST, Hansen MA, Schroeder TV, et al. Endovascular treatment of hemodialysis arteriovenous fistulas: is immediate post-interventional blood flow a predictor of patency. J Vasc Access 2012;13:315-20.

23. Neuen BL, Gunnarsson R, Webster AC, et al. Predictors of patency after balloon angioplasty in hemodialysis fistulas: a systematic review. J Vasc Interv Radiol 2014;25:917-24.

24. Miyamoto K, Sato T, Momohara K, et al. Analysis of factors for post-percutaneous transluminal angioplasty primary patency rate in hemodialysis vascular access. J Vasc Access 2020;21:892-9.

25. Aktas A, Bozkurt A, Aktas B, et al. Percutaneous transluminal balloon angioplasty in stenosis of native hemodialysis arteriovenous fistulas: technical success and analysis of factors affecting postprocedural fistula patency. Diagn Interv Radiol 2015;21:160-6.

26. Wu CC, Wen SC, Yang CW, et al. Baseline plasma glycemic profiles but not inflammatory biomarkers predict symptomatic restenosis after angioplasty of arteriovenous fistulas in patients with hemodialysis. Atherosclerosis 2010;209:598-600.

27. Liu JH, Lin PW, Liu YL, et al. Comparison of classical and non-classical cardiovascular risk factors influencing the

Cite this article as: Zheng Q, Xie B, Xie X, Zhang W, Hou J, Feng Z, Tao Y, Yu F, Zhang L, Ye Z. Predictors associated with early and late restenosis of arteriovenous fistulas and grafts after percutaneous transluminal angiography. Ann Transl Med 2021;9(2):132. doi: 10.21037/atm-20-7690 patency of native arteriovenous fistulas after percutaneous transluminal angioplasty therapy among haemodialysis patients. Postgrad Med J 2007;83:547-51.

28. Doi S, Masaki T, Shigemoto K, et al. Calcium channel antagonists reduce restenosis after percutaneous transluminal angioplasty of an arteriovenous fistula in hemodialysis patients. Ther Apher Dial 2008;12:232-6.

29. Chen FA, Chien CC, Chen YW, et al. Angiotensin Converting-Enzyme Inhibitors, Angiotensin Receptor Blockers, and Calcium Channel Blockers Are Associated with Prolonged Vascular Access Patency in Uremic Patients Undergoing Hemodialysis. PLoS One 2016;11:e0166362.

30. Wu CC, Wen SC, Yang CW, et al. Plasma ADMA predicts restenosis of arteriovenous fistula. J Am Soc Nephrol 2009;20:213-22.

31. Clark TW, Hirsch DA, Jindal KJ, et al. Outcome and prognostic factors of restenosis after percutaneous treatment of native hemodialysis fistulas. J Vasc Interv Radiol 2002;13:51-9.

32. Zhu ZR, Zou L, Xing Y, et al. Predictors of primary patency after percutaneous balloon angioplasty for stenosis of Brescia-Cimino hemodialysis arteriovenous fistula. Br J Radiol 2020;93:20190505.

33. Wakamoto K, Doi S, Nakashima A, et al. Comparing the 12-month patency of low-versus high-pressure dilation in failing arteriovenous fistulae: A prospective multicenter trial (YOROI study). J Vasc Access 2018;19:477-83.

34. Lin SJ, Tung CW, Hsu YC, et al. The Prognostic Significance of Puncture Timing to Survival of Arteriovenous Fistulas in Hemodialysis Patients: A Multicenter Retrospective Cohort Study. J Clin Med 2019;8:247.

(English Language Editor: C. Betlazar-Maseh) 